\title{
Moral Harassment and Resilience on the Medical Formation: a Study With First Year Students
}

\author{
Marcelo G. Lima; Ana B. Tokeshi; Liliam A. Fernandes; Thiago M. Fidalgo
}

Background: Moral harassment - a global problem rooted in medical culture - has a negative impact on medical formation. Resilience, conversely, is a protective competence against adverse situations, including harassment. Although they are relevant topics, there are few studies about moral harassment and resilience in medical formation.

Objectives: The objective is to obtain data about resilience and moral harassment, which are part of the mental health of medical students and compound ethical-professional formation of them.

Materials and Methods: Data collection was done during the enrollment of the students in the first year at Universidade Federal de São Paulo in February 2019. The data collection followed 4 questionnaires: 1) Sociodemographics; 2) Patient Health Questionnaire-4; 3) Wagnild-Young Resilience Scale- 25 items $\AA$; 4) Questionnaire about moral harassment in previous moments of life.

\section{Questionnaire about moral harassment in previous moments of life $n=107$}

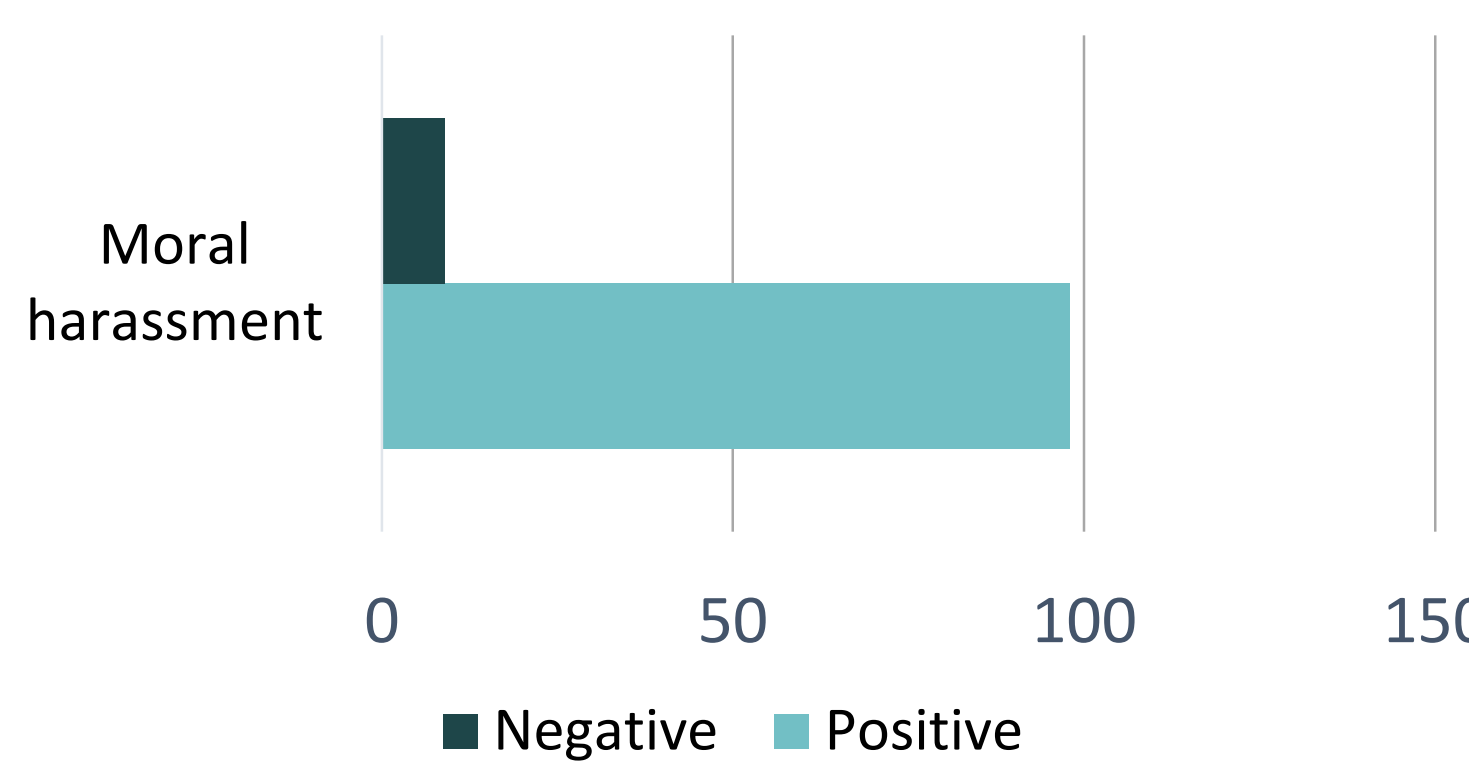

Patient Health Questionnaire-4 (PHQ-4) n=107

Suggestive of Depression and Anxiety

Suggestive of Anxiety

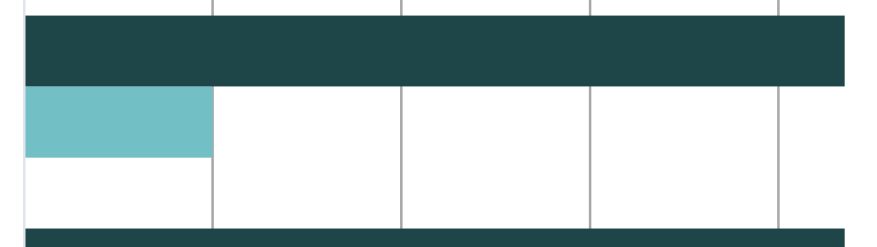

Suggestive of Anxiety

Suggestive of Depression

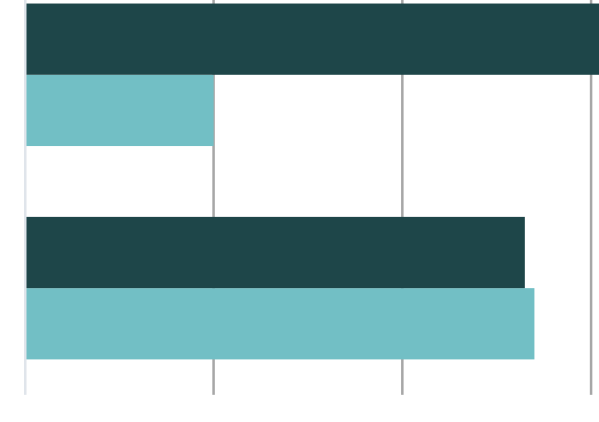

20

40

60

80

Negative Positive
Results: All questionnaires had 109 responses, which represent a $90 \%$ of all first-year students. The main findings were: i) $91 \%$ of participants reported that they have suffered some type of moral harassment before; ii) $55 \%$ of participants are on the lower scores of the Resilience Scale: "Very low" and "Low"; iii) $63 \%$ of students had non-normal scores on PHQ-4 tracking; iv) $49 \%$ of students had suggestive levels of depression, 19\% suggestive levels of anxiety, and 19\% suggestive levels of depression and anxiety on $\mathrm{PHQ}-4$ tracking.

Discussion: Around the world, some studies suggested that on the enrollment medical students were as satisfied with their lives as other students. But, at the end of the first year, medical students were less satisfied in comparison with the other ones. Further, there are some studies which reveal that establishing horizontal relationships with colleagues, senior students and professors is the first step on the prevention of moral harassment.

Conclusions: These data provides some worrying notes: while the moments of moral harassment, depression and anxiety have high levels among students, the resilience, a protective competence, have low levels. This reinforces the need for implementation of preventive-interventive strategies about medical education.

Wagnild-Young Resilience Scale $n=107$

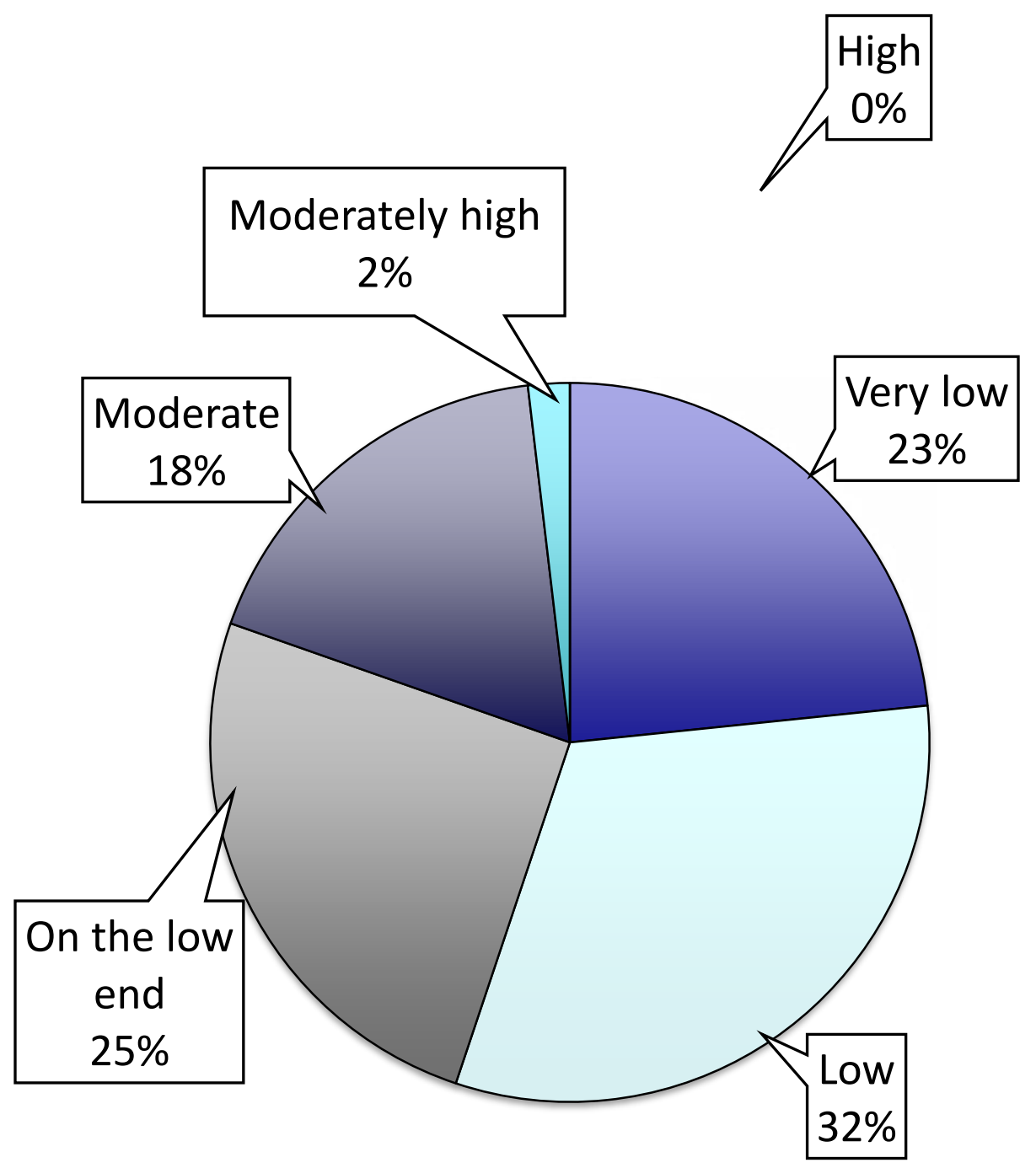

\section{References:}

- Hirigoyen, MF. Mal-estar no trabalho: redefinindo o assédio moral. Rio de Janeiro: Betrand Brasil, 2002. - Löwe B et al. A 4-item measure of depression and anxiety: validation and standadization of the Patient Health Questionnaire-4 (PHQ-4) in the general population. J Affect Disord. 2010; 122:86-95.

- Wagnild G, Young H. Development and psychometric evaluation of teh Resilience Scale. Journal of Nursing Measurement. 1993; 1(2): 165-178. 